\title{
Mammary tumor modifiers in BALB/cJ mice heterozygous for p53
}

\author{
Joanna G. Koch · Xiangjun Gu • Younghun Han · Adel K. El-Naggar • \\ Melissa V. Olson · Daniel Medina · D. Joseph Jerry · Anneke C. Blackburn • \\ Gary Peltz $\cdot$ Christopher I. Amos $\cdot$ Guillermina Lozano
}

Received: 30 November 2006/ Accepted: 9 April 2007/Published online: 8 June 2007

(C) Springer Science+Business Media, LLC 2007

\begin{abstract}
BALB} / \mathrm{c}$ mice are predisposed to developing spontaneous mammary tumors, which are further increased in a $p 53$ heterozygous state. $\mathrm{C} 57 \mathrm{BL} / 6 \mathrm{~J}$ mice are resistant to induced mammary tumors and develop less than $1 \%$ mammary tumors in both wild-type and $p 53^{+/-}$states. To map modifiers of mammary tumorigenesis, we have established $\mathrm{F}_{1}$ and $\mathrm{F}_{2}$ crosses and backcrosses to BALB/cJ (N2-BALB/cJ) and C57BL/6J (N2-C57BL/6J) strains. All cohorts developed mammary carcinomas in $p 53^{+/-}$females, suggesting that multiple loci dominantly and recessively contributed to mammary tumorigenesis. We mapped two modifiers of mammary tumorigenesis in the BALB/cJ
\end{abstract}

Electronic supplementary material The online version of this article (doi: 10.1007/s00335-007-9028-2) contains supplementary material, which is available to authorized users.

\section{J. G. Koch · M. V. Olson · G. Lozano}

The University of Texas Graduate School of Biomedical Sciences and the Department of Cancer Genetics,

The University of Texas M. D. Anderson Cancer Center, Houston, Texas 77030, USA

X. Gu $\cdot$ Y. Han $\cdot$ C. I. Amos

Department of Epidemiology,

The University of Texas M. D. Anderson Cancer Center, Houston, Texas 77030, USA

\author{
A. K. El-Naggar \\ Department of Pathology, \\ The University of Texas M. D. Anderson Cancer Center, \\ Houston, Texas 77030, USA \\ D. Medina \\ Department of Molecular and Cellular Biology, \\ Baylor College of Medicine, Houston, \\ Texas 77030, USA
}

strain. Mtsml (mammary tumor susceptibility modifier), a dominant-acting modifier, is located on chromosome 7. $M t s m 1$ is suggestive for linkage to mammary tumorigenesis $(p=0.001)$. We have analyzed the Mtsml region to locate candidate genes by comparing it to a rat modifier region, $M c s 3$, which shares syntenic conservation with Mtsml. Expression data and SNPs were also taken into account. Five potential candidate genes within Mtsml are Aldhla3, Chd2, Nipa2, Pcsk6, and Tubgcp5. The second modifier mapped is $M t s m 2$, a recessive-acting modifier. Mtsm2 is located on chromosome $\mathrm{X}$ and is significantly linked to mammary tumorigenesis $\left(p=1.03 \times 10^{-7}\right)$. 


\section{Introduction}

Breast cancer is the most frequent type of cancer in women in the United States (Wingo et al. 1998). Several genes have been identified that, when inherited, increase a woman's likelihood of developing breast cancer. These include, but are not limited to, BRCA1, BRCA2, PTEN, and TP53 and account for only approximately $5 \%-10 \%$ of breast cancers (Balmain et al. 2003).

Most patients with Li-Fraumeni syndrome (LFS) inherit TP53 mutations (Malkin et al. 1990; Srivastava et al. 1990). LFS patients develop many types of tumors, including soft tissue sarcomas, osteosarcomas, breast carcinomas, and brain tumors, with breast carcinomas being the most frequent tumor type in women (Evans and Lozano 1997; Kleihues et al. 1997). Different patients within a family with the same TP53 mutation develop different types of cancers. While a majority of LFS patients develop cancer at a young age, the latency of tumorigenesis also varies widely within families. The latency and occurrence of LFS-related cancers are affected by environmental causes such as smoking (Hwang et al. 2003). Generation or birth cohort effects can also affect latency of breast cancer in LFS patients (Brown et al. 2005; Trkova et al. 2002). Thus, additional genes, or modifiers, may predispose patients to a particular type of tumor or modify the latency of that tumor. These modifiers may also be present in the general population and could contribute to "spontaneous" breast cancer (Balmain et al. 2003).

Recently, a modifier of cancer risk in Li-Fraumeni syndrome was discovered. $M d m 2$ encodes a negative regulator of p53 and contains a single nucleotide polymorphism (SNP) that alters a $\mathrm{T}$ to $\mathrm{G}$ at nucleotide 309 in the promoter region of Mdm2 (Bond et al. 2004). SNP309 causes an increase in $M d m 2$ RNA and protein levels, which in turn decreases p53 activity. SNP309 has been associated with an earlier median age of breast cancer onset in women with Li-Fraumeni syndrome, with breast cancer occurring ten years earlier in patients with $\mathrm{G} / \mathrm{T}$ or $\mathrm{G} / \mathrm{G}$ genotypes. A similar decrease in latency was seen in patients with no evidence of an inherited predisposition to cancer (Bond et al. 2004, 2005).

To model the Li-Fraumeni syndrome, mice with $p 53$ deletions have been made on three different inbred strains. Each strain shares many similarities in tumor development; all three strains develop sarcomas, lymphomas, and some carcinomas. However, each strain also develops a unique tumor type. The 129Sv strain develops teratomas (Harvey et al. 1993), while the BALB/cMed strain develops mammary carcinomas (Kuperwasser et al. 2000), both of which are rarely seen in the C57BL/6J strain (Donehower et al. 1992, 1995; Harvey et al. 1993; Jacks et al. 1994; Kuperwasser et al. 2000). Harvey et al. (1993) suggested that the $129 \mathrm{~Sv}$ strain was already predisposed to tumors, specifically teratomas, and that loss of p53 only served to accelerate the occurrence of these tumors. They also suggested that genetic background plays a role in both the type of tumor that develops and tumor latency. Indeed, wild-type $129 \mathrm{~Sv}$ mice develop teratomas, and wild-type BALB/c mice develop mammary tumors, albeit at low levels with long latency (Altman and Katz 1979; Heston and Vlahakis 1971; Kuperwasser et al. 2000). Loss of $p 53$ in both strains decreases the latency and increases the occurrence of these tumor types in their respective strains (Harvey et al. 1993; Kuperwasser et al. 2000). Differences in tumor development between mouse strains can be exploited to map modifying, low-penetrance genes involved in specific tumor types and possibly be extrapolated to patients with Li-Fraumeni syndrome.

$\mathrm{BALB} / \mathrm{c}$ mice have been used to study the propensity of mammary tumorigenesis. Twenty percent of $B A L B / c$ mice develop spontaneous mammary tumors at an average latency of 16.7 months (Heston and Vlahakis 1971). $\mathrm{BALB} / \mathrm{cMed}$ mice that are heterozygous for $p 53$ have increased incidence of mammary tumors at $42 \%$ in a cohort of males and females (Kuperwasser et al. 2000). In a cohort with only virgin female BALB/cMed $p 53^{+/-}$mice, $65 \%$ develop mammary tumors with a latency of $8-14$ months (Blackburn et al. 2003; Kuperwasser et al. 2000). C57BL/6 mice develop less than $1 \%$ mammary tumors in both the wild-type and $p 53^{+/-}$states (Heston and Vlahakis 1971; Jacks et al. 1994). Blackburn et al. (2003) have shown that $\mathrm{F}_{1}$ mice with 50\% C57BL/6 and 50\% BALB/c background developed mammary tumors at a lower incidence (32\%) than BALB/c mice, and that $\mathrm{N}_{2}$ mice with $75 \% \mathrm{BALB} / \mathrm{c}$ background developed an intermediate level of mammary tumors $(45 \%)$. These data suggest that both dominant and recessive modifiers are involved in the BALB/c susceptibility to mammary tumors. However, no mapping data are available from these studies.

We have chosen to map modifiers that increase the occurrence and decrease latency of mammary tumors in mice. We crossed the C57BL/6J (resistant) and BALB/cJ (susceptible) strains and followed inheritance of the mammary tumor phenotype with inheritance of alleles from the BALB/c strain to map regions of the genome that are likely to contain modifiers that increase susceptibility to mammary tumorigenesis. We have located two potential modifiers of mammary tumorigenesis in the BALB/cJ strain. One locus, named Mtsml (mammary tumor susceptibility modifier 1) is on chromosome 7 , and a second locus, named Mtsm2, is located on chromosome X. 


\section{Method}

Mice and tumors

C57BL/6J $p 53^{+/-}$(formally named B6.129S2-Trp53 $3^{\text {tm } 17 y j} / \mathrm{J}$ ) and BALB/cJ mice were purchased from The Jackson Laboratory (Bar Harbor, ME). The mice were housed in a conventional specific pathogen-free colony with a $12-\mathrm{h}$ light cycle and were fed ad libitum. Genotyping for $p 53$ was performed as previously described (Jacks et al. 1994). The mice were monitored for tumors daily and were sacrificed when a tumor was visible or the mouse was moribund. A small section of each tumor was fixed in $10 \%$ buffered formalin, and any remaining sections of tumors were frozen and stored at $-80^{\circ} \mathrm{C}$. The fixed tissues were paraffin embedded, sectioned, and stained with hematoxylin and eosin in the histology core facility at M.D. Anderson Cancer Center. All mouse protocols were approved by the IACUC.

\section{Marker analysis}

ABI Prism Mouse Mapping Primers (Applied Biosystems, Foster City, CA) were selected using the Center for Inherited Disease's software tool for STRP mapping panels (http://www.cidr.org). The centimorgan location of these markers was taken from The Jackson Laboratory's Mouse Genome Informatics website (http://www.informatics.jax.org), while megabase locations were taken from Ensembl version 38 (http://www.ensembl.org). Each marker was verified for polymorphic PCR amplification using genomic DNA from BALB/cJ and C57BL/6J wild-type mice.

Genomic DNA from each mouse was extracted from tail biopsies at weaning and/or normal liver tissues after sacrifice. PCR was performed separately for each mouse and primer. The PCR products were then multiplexed and run on an ABI 3100 16-capillary genetic analyzer to detect the polymorphic size differences. Data were analyzed using Applied Biosystem's Genotyper 3.5 NT program.

Additional markers for fine mapping were selected from the Whitehead Institute/MIT Center for Genome Research Genetic Maps (http://www.broad.mit.edu/cgibin/mouse/index) and purchased from Research Genetics (MapPairs primers). PCR for most of these markers was performed as follows: $95^{\circ} \mathrm{C}$ for $1 \mathrm{~min} ; 35$ cycles of $95^{\circ} \mathrm{C}$ for $30 \mathrm{sec}, 55^{\circ} \mathrm{C}$ for $30 \mathrm{sec}, 72^{\circ} \mathrm{C}$ for $45 \mathrm{sec}$; followed by $72^{\circ} \mathrm{C}$ for 5 min. Markers D7Mit321 and D7Mit353 required an annealing temperature of $59^{\circ} \mathrm{C}$. The products were run on a $4 \%$ NuSieve 3:1 agarose gel (Cambrex, East Rutherford, NJ).
Statistical analysis

Prior to statistical analysis the markers were aligned according to megabase positions along the chromosomes, based on Ensembl version 38. Any markers that showed double recombination with flanking markers were flagged and regenotyped to confirm results. We then used KaplanMeier survival analysis to assess the relationship between time to onset for mammary tumors and the genotypes at each marker. To assess significance with the Kaplan-Meier analyses, we used a log rank test. To evaluate the joint effects of both markers on time to onset for cancer, we used Cox proportional hazards models and tested for significance using a Wald test. To evaluate evidence for interactions, we applied Cox proportional hazards models that included interaction terms among SSLPs on chromosomes $\mathrm{X}$ and 7 that were found in univariable analyses to significantly predict time to onset for mammary tumors. To evaluate the dominance or recessivity of alleles at the chromosome $\mathrm{X}$ and 7 loci, we used Cox regression analysis to compare the hazard ratios for the homozygous animals to those of the heterozygous animals. Under the recessive model, the hazard for mice homozygous for C57BL/6J alleles equals the hazard for heterozygous animals, while the BALB/cJ homozygous mice would have a higher hazard ratio. Under a dominant model, the homozygous $\mathrm{BALB} / \mathrm{cJ}$ and heterozygous mice have similar hazards that are higher than those of the C57BL/6J homozygous mice. All analyses were conducted using STATA version 8 (Stata Corporation, College Station, TX). Of the 75 animals genotyped for the entire genome, 55 were $\mathrm{F}_{2}$ mice, 13 were $\mathrm{N}_{2}$ C57BL/6J, and 7 were $\mathrm{N}_{2}$ BALB/cJ. Some analyses were restricted to include only $F_{2}$ intercross animals to insure similar results to our mixed cohort study. Eighty-six mice, which included ten additional $\mathrm{F}_{2}$ and one $\mathrm{N}_{2}$ BALB/ cJ mice with mammary tumors, were genotyped for D7Mit91, D7Mit201, D7Mit350, and DXMit68.

\section{Candidate gene selection}

The rat modifier Mcs3 (Shepel et al. 1998) was defined using Ensembl v38 (http://www.ensemble.org) and the Rat Genome Database release 7.7 (http://www.rgd.mcw.edu). The rat modifier is surrounded by D1Mit11 and DlMit2 at 102,532,145 and 134,980,527 bp. SoftBerry's (http:// www.softberry.com/berry.phtml) Rat-Mouse Synteny for chromosome 1 of rat was used to determine regions of syntenic conservation between rat and mouse in the Mcs3 region. Gene names and abbreviations, if not listed directly on SoftBerry, were determined by searching Ensembl (mouse and/or rat versions), Mouse Genome Informatics (http://www.informatics.jax.org) (Eppig et al. 2005), or 
NCBI (http://www.ncbi.nlm.nih.gov) using other information provided by SoftBerry, and confirmed by position on the rat and/or mouse chromosomes in Ensembl.

Single nucleotide polymorphisms (SNPs) between BALB/cJ and C57BL/6J strains in the Mtsml region (27.4$71.8 \mathrm{Mb}$ ) were identified using the "Strains and Polymorphisms" link on The Jackson Laboratory's Mouse Genome Informatics website (http://www.informatics.jax.org), which is based on NCBI dbSNP build 125 .

\section{Expression data}

The fourth inguinal mammary glands, with the intramammary lymph nodes removed, were collected from 12-weekold virgin C57BL/6J $p 53^{+-}$and BALB/cMed $p 53^{+-}$mice and snap-frozen in liquid nitrogen. Total RNA was reversetranscribed using a T7 promoter-coupled oligo(d)T primer [GeneChip T7-Oligo(d)T Promoter Primer Kit, Affymetrix, Santa Clara, CA]. After the second-strand cDNA synthesis, an in vitro transcription reaction was performed using an Enzo BioArray High Yield RNA transcript labeling kit (Affymetrix). The labeled samples were hybridized to the Murine Genome U74v2 set that contains probe sets for approximately 36,000 full-length mouse genes and EST clusters from the UniGene database (Affymetrix). GeneChips were scanned using the GS2500 scanner and images were analyzed by Affymetrix software (Microarray Analysis Suite version 5.0). Four mice of each strain were analyzed with pairwise comparisons. Genes showing at least 1.5 -fold expression differences, with intensity values greater than 150 in one strain and $p<0.05$ were considered to be differentially expressed.

\section{Real-time RT-PCR}

The fourth inguinal mammary glands with the intramammary lymph nodes removed were isolated from 3-12-weekold $\mathrm{C} 57 \mathrm{BL} / 6 \mathrm{~J}$ and BALB/cJ wild-type animals and snapfrozen in liquid nitrogen. Total RNA was extracted using the Qiagen RNeasy kit (Qiagen, Valencia, CA) and reversetranscribed using a T7 promoter-coupled oligo(d)T primer (First Strand cDNA Synthesis Kit, Amersham Biosciences, Piscataway, NJ). The primer sequences for Gapdh were previously described (Iwakuma et al. 2004). The following primers sets were used: Aldhla3, TGTGTGGACAGATCATCCCG and TCCAGGTGAACATCAGCAGG, $C h d 2$, CCTGTGGACCCTGAGGAAAA and TTTGACTC GGCAGGTTAGGC, Nipa2, GTGGGCTGGACTGCTGTCA and GCAGCAAAATTGGCCACTTC, Pcsk6, AGCAATGCCGATGAGACCTT and GCAGAGCCGATTGG ACTTCA, and Tubgcp5, TGTTGGCCATCAACTTTGCA and CGTGAAAGTCGCTCTGCTCC. Each sample was analyzed in triplicate and expression of each target gene was normalized to expression of Gapdh in that reaction. Fold change was determined by dividing the mean of the three C57BL/6J animals (in triplicate) by the BALB/cJ mean for each animal.

\section{Results}

Previous studies using BALB/c $p 53^{+/-}$mice have indicated an increased susceptibility of BALB/c mice to mammary tumors compared with C57BL/6J mice (Blackburn et al. 2003; Kuperwasser et al. 2000). We therefore set up four cohorts of mice to map mammary tumor modifiers in the BALB/cJ strain. B6.129S2-Trp53tm1Tyj/J mice containing a $p 53$ null allele in the $\mathrm{C} 57 \mathrm{BL} / 6 \mathrm{~J}$ background (called C57BL/6J I $p 53^{+/-}$in this study) were crossed with BALB/ cJ mice to create an $F_{1}$ cohort of 58 female $p 53^{+/-}$mice. $F_{1}$ mice were then crossed to create an $F_{2}$ cohort of 155 female $p 53^{+/-}$mice. Two backcross cohorts were also established by crossing $\mathrm{F}_{1}$ mice to $\mathrm{BALB} / \mathrm{cJ}$ mice to create an $\mathrm{N}_{2}-\mathrm{BALB} / \mathrm{cJ}$ cohort with 50 female $p 53^{+/-}$mice and to C57BL/6J mice to create an $\mathrm{N}_{2}-\mathrm{C} 57 \mathrm{BL} / 6 \mathrm{~J}$ cohort with 37 female $p 53^{+/-}$mice.

All cohorts developed the expected types of tumors, including lymphomas, sarcomas, and some carcinomas (Table 1). In addition, glandular and cystic hyperplasias of the uterus and ovaries were found in our cohorts that have not been previously reported in any p53 mouse model. All cohorts developed mammary tumors, which were primarily adenocarcinomas with some adenosquamous carcinomas. In addition, we observed increased mammary tumor development and decreased latency in mice with a greater percentage of BALB/cJ alleles (Table 2). In the $\mathrm{F}_{1}$ cohort (50\% BALB/cJ and 50\% C57BL/6J alleles), $28 \%$ of the females developed mammary tumors with a median latency of 489 days. The presence of mammary tumors in the $F_{1}$ cohort indicates the presence of at least one dominant mammary tumor susceptibility modifier in the BALB/cJ background. In the $\mathrm{N}_{2}-\mathrm{BALB} / \mathrm{cJ}$ cohort $(75 \% \mathrm{BALB} / \mathrm{cJ}$ and $25 \%$ C57BL/6J alleles), $40 \%$ of the females developed mammary tumors with a median latency of 391 days. The increased number of mammary tumors and decreased latency in the $\mathrm{N}_{2}$-BALB/cJ cohort, compared to the $\mathrm{F}_{1}$ cohort, indicates the presence of recessive modifiers for mammary tumorigenesis in the BALB/cJ strain.

In the initial mapping study, 75 virgin female mice, including 28 with mammary tumors and 47 without mammary tumors, were genotyped with 166 microsatellite markers across the genome. Fifty-five of these mice were from the $F_{2}$ cohort (19 with mammary tumors), with the remaining 20 from backcross cohorts $\left[13 \mathrm{~N}_{2}\right.$ C57BL/6J (4 with mammary tumors) and $7 \mathrm{~N}_{2} \mathrm{BALB} / \mathrm{cJ}$ (5 with mammary tumors)]. The markers are ABI Prism Mouse 
Table 1 Tumors in mixed background $p 53^{+/-}$females

\begin{tabular}{lllll}
\hline Tumor types & $\mathrm{F}_{1}$ & $\mathrm{~F}_{2}$ & $\mathrm{~N}_{2}$-BALB/cJ & $\mathrm{N}_{2}-\mathrm{C} 57 \mathrm{BL} / 6 \mathrm{~J}$ \\
\hline Carcinomas & $36 \%(23)^{\mathrm{a}}$ & $26 \%(43)$ & $43 \%(24)$ & $13 \%(5)$ \\
Mammary & $25 \%(16)$ & $18 \%(30)$ & $36 \%(20)$ & $11 \%(4)$ \\
Lung & $8 \%(5)$ & $5.5 \%(9)$ & $5 \%(3)$ & 0 \\
Other & $3 \%(2)$ & $2.5 \%(4)$ & $2 \%(1)$ & $3 \%(1)$ \\
Sarcomas & $42 \%(26)$ & $46 \%(75)$ & $32 \%(18)$ & $60 \%(22)$ \\
Osteosarcomas & $22 \%(14)$ & $26 \%(43)$ & $16 \%(9)$ & $38 \%(14)$ \\
Angiosarcomas & $10 \%(6)$ & $8 \%(13)$ & $3.5 \%(2)$ & 0 \\
Histiocytic & 0 & $3 \%(4)$ & $3.5 \%(2)$ & $16 \%(6)$ \\
Other & $10 \%(6)$ & $9 \%(15)$ & $9 \%(15)$ & $27 \%(10)$ \\
Lymphomas & $22 \%(14)$ & $28 \%(45)$ & $25 \%(14)$ & 37 \\
Total tumors & 63 & 163 & 56 & 37 \\
Total mice & 58 & 155 & $17 \%(27)$ & $12 \%(6)$ \\
Mice with hyperplasia of uterus and/or ovary & $22 \%(13)$ & & $27 \%(10)$ \\
\hline
\end{tabular}

${ }^{a}$ Number of tumors are shown in parentheses

Table 2 Mammary tumor incidence and latency in female $p 53^{+/-}$mice

\begin{tabular}{lllll}
\hline & $\mathrm{F}_{1}$ & $\mathrm{~F}_{2}$ & $\mathrm{~N}_{2}$-BALB/cJ & $\mathrm{N}_{2}$-C57BL/6J \\
\hline $\begin{array}{lll}\text { Median latency of mammary tumors(days) } \\
\% \text { of mice with mammary tumors }\end{array}$ & 489 & 445 & 391 & $385^{\mathrm{a}}$ \\
\hline
\end{tabular}

${ }^{a}$ Incidence and latency are skewed due to low number of mice in cohort

Fig. 1 Positions of markers on mouse genome used in genomewide modifier screen. Numbers reflect the last number in the marker name. For example, the first marker on chromosome 1 is D1Mit430. Each marker was placed on the chromosome at its relative centimorgan location
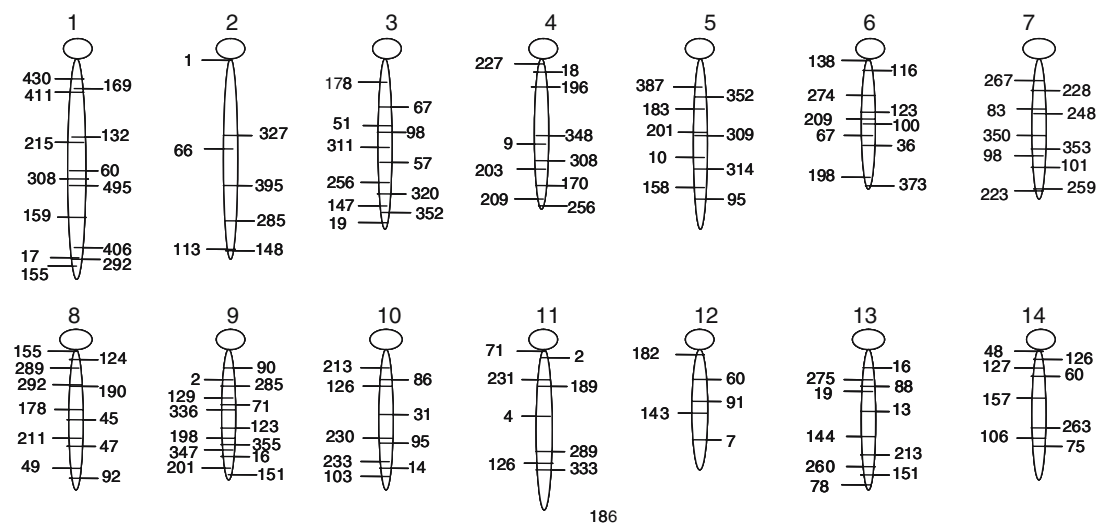
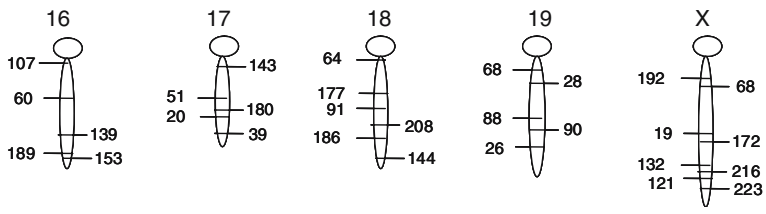

Mapping Primers and are an average of $8.4 \mathrm{cM}$ apart, with the median distance being $6 \mathrm{cM}$. The largest gap is on chromosome 15 from 0 to $40.9 \mathrm{cM}$ at the centromeric end (Fig. 1). The statistical analyses were based on the presence or absence of mammary tumors and time to onset.

Initial mapping of the cohort described above showed that chromosomes $6,7,17,18$, and $\mathrm{X}$ each contained one or more markers with $p<0.05$, suggesting the presence of at least five possible modifiers (Table 3 ). While a $p$ value of 0.05 cannot be reported as definitive evidence for linkage, it has been set by Lander and Kruglyak (1995) as the upper limit of $p$ that should be reported from a complete genome scan. These criteria adjust for inherent multiple testing when analyzing data from the genome-wide scans. On 
Table 3 Log rank tests from survival analyses of markers from genome-wide scan

\begin{tabular}{lccc}
\hline Marker $^{\mathrm{a}}$ & $\mathrm{cM}$ & $\mathrm{Mb}$ & $p$ value \\
\hline D6Mit274 & & & 0.0288 \\
D7Mit83 & 26.5 & 39.0 & 0.0177 \\
D7Mit248 & 27.8 & $\mathrm{~nm}^{\mathrm{b}}$ & 0.0123 \\
D7Mit350 & 41.0 & 70.7 & 0.0140 \\
D17Mit245 & & & 0.0062 \\
D18Mit144 & & & 0.0474 \\
DXMit192 & 16.0 & 45.4 & 0.0217 \\
DXMit68 & 17.2 & 45.8 & 0.00004 \\
\hline
\end{tabular}

${ }^{a}$ A total of 75 mice were genotyped at 166 markers, $55 \mathrm{~F}_{2}$ of which 19 had mammary tumors, $13 \mathrm{~N}_{2}$-C57BL/6J of which 4 had mammary tumors, and $7 \mathrm{~N}_{2}$-BALB/cJ of which 5 had mammary tumors

${ }^{\mathrm{b}} \mathrm{nm}=$ not mapped to Ensembl

chromosome 7, initial mapping indicated that several markers had $p<0.05$ (Table 3). We then focused on chromosome 7 and increased the density of the markers by adding eight markers between D7Mit83 and D7Mit321, a region containing three consecutive markers with $p<0.05$. The original 75 mice were genotyped for these new markers, after which D7Mit201 had the lowest $p$ value (0.0025) (Table 4). An additional ten $F_{2}$ mice and one $\mathrm{N}_{2}$-BALB/cJ mouse with mammary tumors, which were also $p 53^{+/-}$virgin females, were genotyped for D7Mit201 and the two flanking markers, D7Mit91 and D7Mit350 (Table 4). These 86 mice included all those with mammary tumors (39) and a comparable number of mice that developed other tumor types (47). The remaining mice in the cohort lacked mammary tumors and were not analyzed. A previous simulation showed no change in relative risks for disease when excluding up to $50 \%$ of observations that were censored at random (Vitezica et al. 2005). Given limitations on genotyping resources and the likely lack of additional statistical significance, we did not test additional mice lacking mammary tumors.

Expanded mapping revealed that marker D7Mit201 was suggestive for linkage to earlier onset of mammary tumorigenesis $(p=0.001)$ (Table 4). Marker D7Mit201 was shown by statistical analysis to be a dominant modifier, which decreases time to onset of mammary tumors (Fig. 2). At the D7Mit201 locus there was no significant difference between heterozygous mice and mice homozygous for $\mathrm{BALB} / \mathrm{cJ}(p=0.14$, hazards ratio $=0.57 \pm 0.224)$,

Table 4 Linkage of markers to mammary tumor susceptibility

\begin{tabular}{|c|c|c|c|c|c|}
\hline \multirow[t]{2}{*}{ Marker $^{\mathrm{a}}$} & \multirow[t]{2}{*}{$\mathrm{cM}$} & \multirow[t]{2}{*}{$\mathrm{Mb}$} & \multicolumn{3}{|l|}{$p$ values } \\
\hline & & & 75 mice $^{b}$ & 86 mice $^{c}$ & $65 \mathrm{~F}_{2}$ mice \\
\hline \multicolumn{6}{|l|}{ Chromosome 7} \\
\hline$D 7 M i t 228^{\mathrm{a}, \mathrm{c}}$ & 18.0 & 27.4 & 0.1049 & & \\
\hline D7Mit82 & 24.0 & 39.0 & 0.0105 & & \\
\hline D7Mit83 & 26.5 & 39.0 & 0.0177 & & \\
\hline D7Mit120 & 26.2 & 41.5 & 0.0164 & & \\
\hline D7Mit86 & 25.1 & 42.9 & 0.0164 & & \\
\hline D7Mit211 & 26.2 & 45.1 & 0.0164 & & \\
\hline D7Mit91 & 27.3 & 46.1 & 0.0164 & 0.0085 & 0.0497 \\
\hline D7Mit201 & 28.4 & 61.4 & 0.0025 & 0.0011 & 0.0069 \\
\hline D7Mit350 & 41.0 & 70.7 & 0.0140 & 0.0261 & 0.1331 \\
\hline D7Mit62 & 31.7 & 71.8 & 0.0317 & & \\
\hline D7Mit301 & 33.9 & 78.8 & 0.0248 & & \\
\hline D7Mit321 & 36.1 & 85.1 & 0.0168 & & \\
\hline$D 7 M i t 353^{\mathrm{a}}$ & 37.2 & 86.8 & 0.0735 & & \\
\hline \multicolumn{6}{|l|}{ Chromosome X } \\
\hline DXMit192 & 16.0 & 45.4 & 0.0217 & & \\
\hline DXMit68 & 17.2 & 45.8 & $4.23 \times 10^{-5}$ & $1.03 \times 10^{-7}$ & $5 \times 10^{-6}$ \\
\hline DXMit $19^{\mathrm{a}}$ & 43.2 & $\mathrm{~nm}$ & 0.1278 & & \\
\hline DXMit $172^{\mathrm{a}}$ & 48.7 & 113.4 & 0.4665 & & \\
\hline
\end{tabular}

$\mathrm{nm}=$ not mapped to Ensembl

${ }^{a}$ Markers are flanking markers with $p>0.05$

b See Table 3 for description of these 75 mice

c This included the original 75 mice and an additional $10 \mathrm{~F}_{2}$ and $1 \mathrm{~N}_{2}-\mathrm{BALB} / \mathrm{cJ}$ mice with mammary tumors 

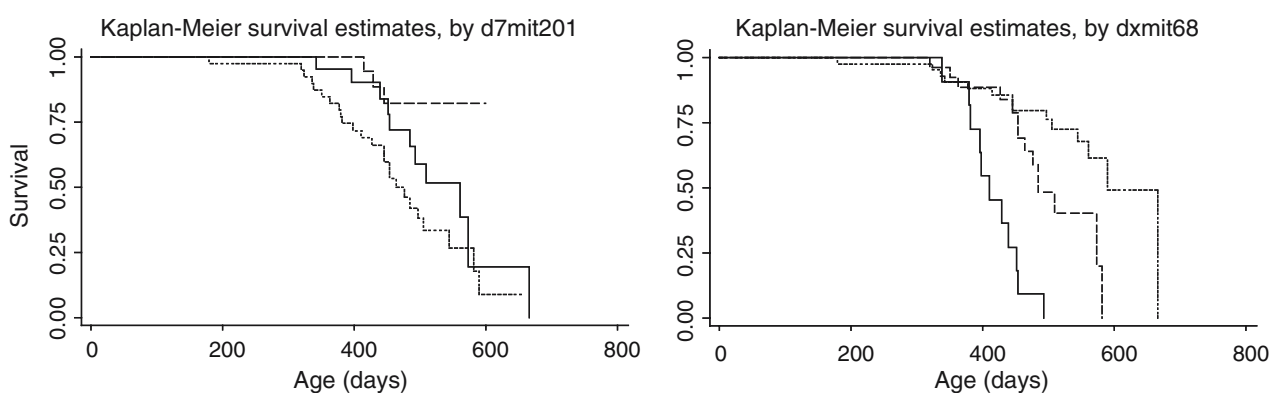

Fig. 2 Kaplan-Meier analyses to assess the relationship between time to onset and genotypes. Eighty-six mice were genotyped with markers D7Mit201 (A) and DXMit68 (B). Dotted lines represent heterozygous mice with one allele of each BALB/cJ and C57BL/6J. Mice homozygous for BALB/cJ alleles are depicted as solid lines, and mice homozygous for C57BL/6J alleles are represented as dashed lines

but heterozygous mice had a higher risk than $\mathrm{C} 57 \mathrm{BL} / 6 \mathrm{~J}$ mice $(p=0.007$, hazard ratio $=4.28 \pm 2.32)$. The surrounding markers D7Mit228 $(p=0.1049)$ and D7Mit353 $(p=0.0735)$ were not significantly linked, or suggestive for linkage, to mammary tumorigenesis. These markers map to 27.4 and $86.8 \mathrm{Mb}$ on chromosome 7 on Ensembl (http://www.ensembl.org). We have named this region Mtsml for mammary tumor susceptibility modifier 1 .

The additional ten $\mathrm{F}_{2}$ mice and one $\mathrm{N}_{2}$-BALB/cJ mouse with mammary tumors were also genotyped for the suggestive marker on chromosome X, DXMit68. After the addition of these mice, marker DXMit68 was statistically significant for linkage to earlier onset of mammary tumorigenesis $\left(p=1.03 \times 10^{-7}\right)$ (Table 4) and was shown to behave primarily as a recessive modifier that decreases time to onset of mammary tumors by statistical analysis (Fig. 2). At the DXMit68 locus there was a minimally significant difference between heterozygous mice and mice homozygous for C57BL/6J $(p=0.025$, hazards ratio $=0.41 \pm 0.16)$, but BALB/cJ mice had a much higher risk than heterozygous mice $(p<0.001$, hazards ratio $=8.14 \pm 3.90)$. No markers closer to the centromere than DXMit68 had $p>0.05$. Moving toward the telomeric end of chromosome $\mathrm{X}$ from DXMit68, the next closest marker, DXMit19 ( $p=0.1278)$, was not significantly linked or suggestive for linkage to mammary tumorigenesis, so the modifier locus is between 0 and $43.2 \mathrm{cM}$. DXMit19 has not been mapped on Ensembl, so the megabase location is unknown. The next closest marker in our mapping panel that has been mapped to Ensembl, which was also not linked to mammary tumorigenesis, was DXMit172, located at $113.4 \mathrm{Mb}$ and $48.7 \mathrm{cM}$. We have named the modifier locus around DXMit68 Mtsm2.

Restriction of the analyses to only $\mathrm{F}_{2}$ intercross animals yielded similar results to those obtained on the entire sample set (Table 4). On chromosome 7 the $p$ values for markers D7Mit91, D7Mit201, and D7Mit350 were 0.05, 0.007 , and 0.13 , respectively. On chromosome $\mathrm{X}$ for marker DXMit68, $p=0.000005$.
There was no evidence of interaction between the loci on chromosomes 7 and $\mathrm{X}$ in influencing time to onset for mammary tumors. The log likelihood model, including only marginal effects from genotypes of D7Mit201 and DXMit68, was 123.52 compared to a log likelihood of 122.18 for a model that included the genotypic effects and all interactions. Comparing the models yields a $\chi^{2}$ test of 2.68 with 4 degrees of freedom, $p=0.61$.

\section{Candidate genes}

The modifier region on mouse chromosome 7, Mtsm1, overlaps a previously mapped rat modifier of mammary carcinoma susceptibility, Mcs3 (Shepel et al. 1998). Mcs3 affects the susceptibility of rats to DMBA-induced mammary carcinomas. We hypothesized that these two modifier regions could contain the same gene or genes responsible for mammary tumorigenesis, whether it develops spontaneously, with loss of $p 53$, or is induced by DMBA. Therefore, we searched for syntenic conservation between rat and mouse genes that were contained in both the Mcs3 and Mtsml modifier regions (Fig. 3). Forty-one genes met this criterion.

To further reduce the list of candidate genes for Mtsm1, we compared the list of conserved genes with expression data. Gene expression data of BALB/cMed $p 53^{+/-}$and

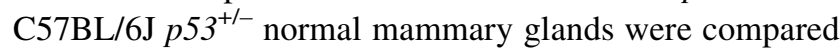
using the Affymetrix Murine U74v2 chip set (Supplementary Table 1). These expression data revealed differential expression of 5 of the 41 conserved genes (Fig. 3). Aldhla3 was expressed 4.8-fold and Pcsk6 was expressed 1.5 -fold higher in C57BL/6J p53 ${ }^{+/-}$mammary glands. Chd2, Nipa2, and Tubgcp5 were expressed 1.7-, 1.5-, and

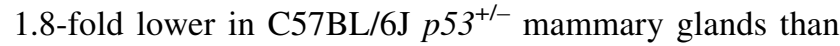
in the BALB/c $p 53^{+/-}$mammary glands. We then searched for known SNPs in these genes between BALB/cJ and C57BL/6J, using the strains and polymorphisms database on The Jackson Laboratory's informatics website (http:// www.informatics.jax.org). Only Pcsk6 contained known 
Fig. 3 Linkage analysis of markers on Aldh1a3, Pcsk6, Tubgcp5, Nipa2, and Chd2, with their location in megabases (in parentheses), are located near D7Mit201, the peak marker in Mtsml. These genes are also located within the rat modifier $M c s 3$, show syntenic conservation between rat and mouse, and are differentially expressed between $\mathrm{BALB} / \mathrm{cMed} p 53^{+/-}$and C57BL/6J $p 53^{+/-}$ mammary glands chromosome 7. Five selected candidate genes,

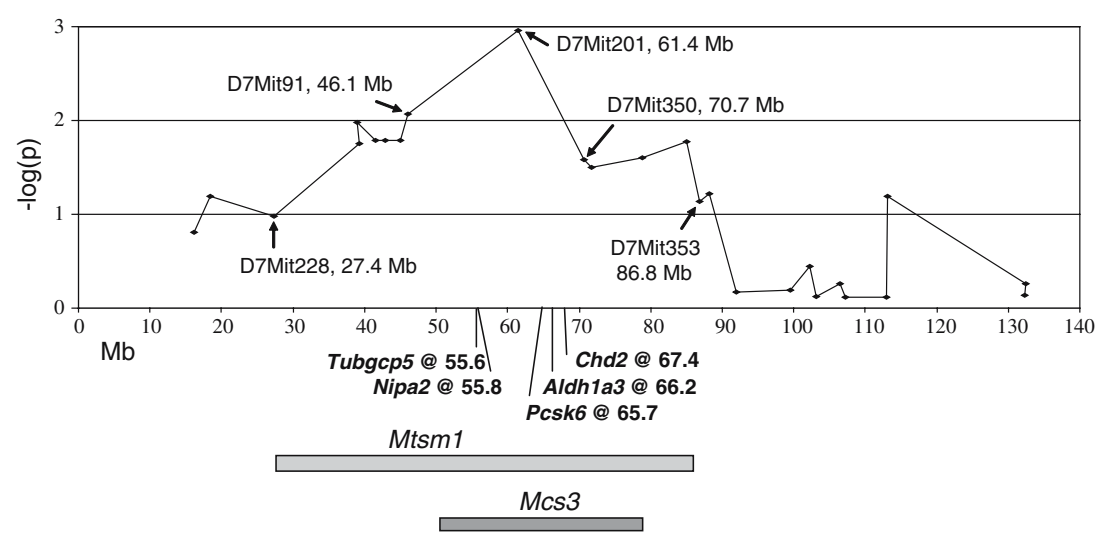

SNPs; one SNP is located in the 3' UTR (untranslated region) (rs4226647) and the second SNP is intronic (rs16805862). To verify the Affymetrix array data, the five genes identified above were analyzed by real-time RT-PCR using mammary glands from BALB/cJ and C57BL/6J wild-type mice. All five genes were differentially expressed and the data replicated the data obtained from Affymetrix arrays (Fig. 4). Based on the evidence presented above, we propose that Aldh1a3, Pcsk6, Chd2, Nipa2, and Tubgcp5 are good candidate genes for Mtsml and should be further examined. Only two genes in this region, cDNA sequence AY078069 and Mfge8, contained coding SNPs. These should also be further examined.

\section{Discussion}

In this study we have characterized crosses between mammary tumor-susceptible (BALB/cJ) and -resistant

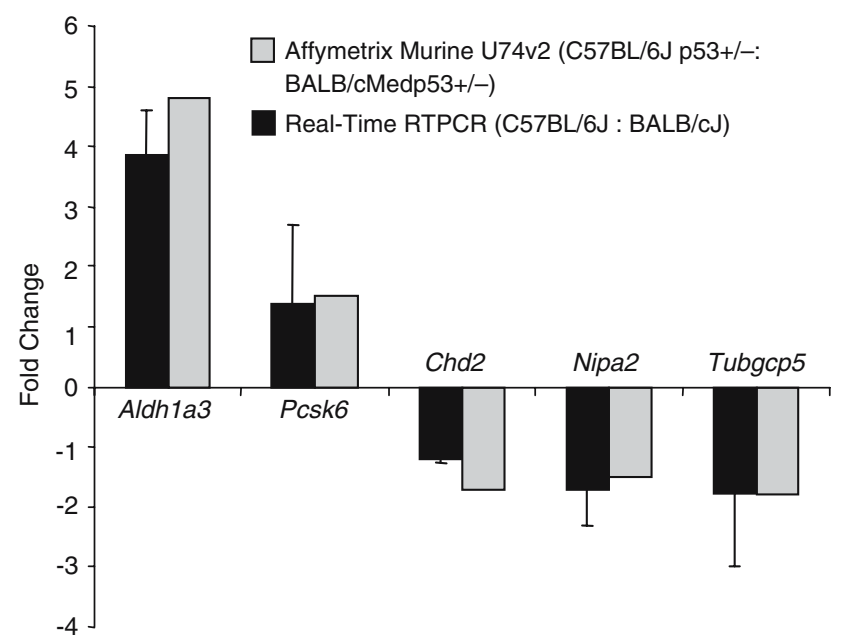

Fig. 4 Differential expression of candidate genes Aldhla3, Pcsk6, Chd2, Nipa2, and Tubgcp5. Bar graphs depict real-time RT-PCR data from C57BL/6J mice compared with BALB/cJ mice (black bars). For comparison, gray bars depict data for the same genes from the Affymetrix Murine Genome U74v2 chip for C57BL/6J $p 53^{+/-}$ compared with the BALB/cMed $p 53^{+-}$strain
(C57BL/6J) $p 53^{+/-}$mice and mapped two potential modifiers. Similar to previously published data (Blackburn et al. 2003), a decrease in mammary tumor incidence in mice with less $\mathrm{BALB} / \mathrm{c}$ alleles and an increase in latency in cohorts with more C57BL/6J alleles were observed. The frequency of mammary tumors was similar in these two studies, with $28 \%$ of our $\mathrm{F}_{1}$ mice developing mammary tumors compared with $32 \%$ of Blackburn's cohort. In addition, $40 \%$ of our $\mathrm{N}_{2}$-BALB/c backcross mice developed mammary tumors compared with $45 \%$ (Blackburn et al. 2003). The latency of the mammary tumors was decreased in our cohorts compared to Blackburn's cohorts. Our $\mathrm{F}_{1}$ cohort developed mammary tumors at a median of 69.8 weeks compared with 74.7 weeks for Blackburn's, and our $\mathrm{N}_{2}-\mathrm{BALB} / \mathrm{c}$ cohort developed mammary tumors at a median of 55.7 weeks compared with 66.4 weeks. While tumor incidence was similar in the two studies, we did not find any pituitary or adrenal gland tumors, while Blackburn's cohorts developed $14 \%$ and $11 \%$ adrenal gland tumors and $2 \%$ and $11 \%$ pituitary tumors in the $\mathrm{N}_{2}$-BALB/c backcross and $F_{1}$ backcross, respectively. We also found in our cohorts that have not been previously reported in any p53 mouse model.

A possible explanation for these differences is that subtle modifications in the $\mathrm{BALB} / \mathrm{c}$ substrains exist. Blackburn's cohorts were created with a substrain of $\mathrm{BALB} / \mathrm{c}$ mice, BALB/cMed. These mice have been bred at Baylor College of Medicine for many years specifically for use in mammary tumor studies. We believe the BALB/cJ and $\mathrm{BALB} / \mathrm{cMed}$ mice may harbor some similar modifiers of mammary tumorigenesis. However, latencies in the two strains are not the same, so each substrain may harbor some unique modifiers of mammary tumor latency.

From our crosses two potential loci for mammary tumor modifiers in the BALB/cJ strain have been detected. One locus on chromosome 7 near marker D7Mit201, designated Mtsml, is a dominant-acting modifier. This modifier overlaps $M c s 3$, a rat modifier of mammary carcinomas. Aldhla3, Pcsk6, Chd2, Nipa2, and Tubgcp5 are likely glandular and cystic hyperplasias in the uterus and ovaries 
candidate genes in Mtsml because they are located within both Mtsml and Mcs3 and are differentially expressed in mammary glands of BALB/cMed $p 53^{+/-}$and C57BL/6J $p 53^{+/-}$mice. Pcsk6 also contains two known SNPs between these strains.

Two genes were upregulated in C57BL/6J mice. Aldhla3, aldehyde dehydrogenase family 1 subfamily a3 (ALDH6 in humans), is necessary for oxidation of retinol to retinoic acid. Many breast cancer cell lines lack the ability to oxidize retinol to retinoic acid, and MCF-7 cells in particular lack expression of ALDH6 (Mira et al. 2000; Rexer et al. 2001). Pcsk6 (PACE4 in humans), proprotein convertase subtilisin/kexin type 6 , cleaves precursor proteins such as hormones, adhesion molecules, growth factors, receptors, and matrix metalloproteinases into their active forms. In human breast cancer tissues, correlation has been found between PACE4 expression and estrogen receptor content (Cheng et al. 1997).

Three genes showed decreased expression in C57BL/6J mice. Tubgcp5, tubulin gamma complex associated protein 5 , is a subunit of the tubulin gamma complex (Murphy et al. 2001), which nucleates microtubulin polymerization during mitosis. Nipa2, nonimprinted in Prader-Willi/Angelman syndrome 2, is a relatively unknown gene (Chai et al. 2003). CHD2, chromodomain helicase DNA binding protein 2, contains a chromatin organization modifier domain near the $\mathrm{N}$ terminus and a SNF2-related helicase/ATPase domain near the center of the protein (Woodage et al. 1997). Any of these genes could be involved in mammary tumorigenesis and will be further studied in this model.

A second locus on chromosome $\mathrm{X}$ near marker DXMit68, designated Mtsm2, is a recessively-acting modifier of mammary tumorigenesis. Mtsm2 is highly significant for mammary tumorigenesis. Timp1, Pnck, and Ar are genes in this modifier region that are involved in mammary growth and/or differentiation that could be potential candidate genes. The androgen receptor $(A r)$ was also expressed 1.49-fold higher in the C57BL/6J $p 53^{+/-}$mammary glands than in BALB/cMed. The androgen receptor has already been implicated in an increased breast cancer incidence and decreased latency in BRCA1-related patients (Rebbeck et al. 1999).

Mtsml and Mtsm2 are linked to mammary tumorigenesis in the BALB/cJ strain, but these are probably not the only mammary tumor modifiers present. Mtsm1 and Mtsm2 did not account for all of the mammary tumors seen in our model, as 3 of the 39 total mice with mammary tumors genotyped did not have any $\mathrm{BALB} / \mathrm{c}$ alleles present at the dominant modifier locus on chromosome 7 or homozygosity for $\mathrm{BALB} / \mathrm{c}$ at the recessive modifier locus on chromosome X. Another 29 mice that did not develop breast cancer did contain BALB/c alleles at these modifier loci. These mice may have succumbed to other tumor types prior to the onset of breast cancer due to p53 heterozygosity. The complexity and cooperativity of low penetrance modifiers could also contribute to our observations.

While the regions that contain modifier loci Mtsml and Mtsm2 remain large and the data suggest that these are not the only modifiers involved in mammary tumorigenesis, we hope that the mapping of these modifier loci and identification of candidate genes will allow future studies to pinpoint genes in these regions that are involved in mammary tumorigenesis. Pinpointing these genes should aid in finding corresponding modifiers of human breast cancer. The identification of novel genes and pathways will further our understanding of the complex pathways involved in susceptibility to breast cancer both in the general population and in inherited cases such as Li-Fraumeni syndrome.

Acknowledgments These studies were supported by fellowships from the American Legion Auxiliary and Schissler Family Foundation to JGK, the training grants in Molecular Genetics of Cancer (CA009299) UO1 CA-04-002 and PO1 CA34936 to GL, and the Cancer Center Support Grant (CA16672) to M. D. Anderson Cancer Center.

\section{References}

Altman PL, Katz DD (1979) Inbred and genetically defined strains of laboratory animals (Bethesda, MD: Federation of American Societies for Experimental Biology)

Balmain A, Gray J, Ponder B (2003) The genetics and genomics of cancer. Nat Genet 33 Suppl:238-244

Blackburn AC, Brown JS, Naber SP, Otis CN, Wood JT, et al. (2003) $\mathrm{BALB} / \mathrm{c}$ alleles for Prkdc and Cdkn2a interact to modify tumor susceptibility in Trp53+/- mice. Cancer Res 63:2364-2368

Bond GL, Hu W, Bond EE, Robins H, Lutzker SG, et al. (2004) A single nucleotide polymorphism in the MDM2 promoter attenuates the p53 tumor suppressor pathway and accelerates tumor formation in humans. Cell 119:591-602

Bond GL, Hu W, Levine A (2005) A single nucleotide polymorphism in the MDM2 gene: from a molecular and cellular explanation to clinical effect. Cancer Res 65:5481-5484

Brown BW, Costello TJ, Hwang SJ, Strong LC (2005) Generation or birth cohort effect on cancer risk in Li-Fraumeni syndrome. Hum Genet 118:489-498

Chai JH, Locke DP, Greally JM, Knoll JH, Ohta T, et al. (2003) Identification of four highly conserved genes between breakpoint hotspots BP1 and BP2 of the Prader-Willi/Angelman syndromes deletion region that have undergone evolutionary transposition mediated by flanking duplicons. Am J Hum Genet 73:898-925

Cheng M, Watson PH, Paterson JA, Seidah N, Chretien M, et al. (1997) Pro-protein convertase gene expression in human breast cancer. Int J Cancer 71:966-971

Donehower LA, Harvey M, Slagle BL, McArthur MJ, Montgomery CA Jr, et al. (1992) Mice deficient for p53 are developmentally normal but susceptible to spontaneous tumours. Nature 356:215221

Donehower LA, Harvey M, Vogel H, McArthur MJ, Montgomery CA Jr, et al. (1995) Effects of genetic background on tumorigenesis in p53-deficient mice. Mol Carcinog 14:16-22

Eppig JT, Bult CJ, Kadin JA, Richardson JE, Blake JA, et al. (2005) The Mouse Genome Database (MGD): from genes to mice-a 
community resource for mouse biology. Nucleic Acids Res 33:D471-D475

Evans SC, Lozano G (1997) The Li-Fraumeni syndrome: an inherited susceptibility to cancer. Mol Med Today 3:390-395

Harvey M, McArthur MJ, Montgomery CA Jr, Bradley A, Donehower LA (1993) Genetic background alters the spectrum of tumors that develop in p53-deficient mice. FASEB J 7:938-943

Heston WE, Vlahakis G (1971) Mammary tumors, plaques, and hyperplastic alveolar nodules in various combinations of mouse inbred strains and the different lines of the mammary tumor virus. Int J Cancer 7:141-148

Hwang SJ, Cheng LS, Lozano G, Amos CI, Gu X, et al. (2003) Lung cancer risk in germline p53 mutation carriers: association between an inherited cancer predisposition, cigarette smoking, and cancer risk. Hum Genet 113:238-243

Iwakuma T, Parant JM, Fasulo M, Zwart E, Jacks T, et al. (2004) Mutation at p53 serine 389 does not rescue the embryonic lethality in $\mathrm{mdm} 2$ or mdm4 null mice. Oncogene 23:7644-7650

Jacks T, Remington L, Williams BO, Schmitt EM, Halachmi S, et al. (1994) Tumor spectrum analysis in p53-mutant mice. Curr Biol 4:1-7

Kleihues P, Schauble B, zur Hausen A, Esteve J, Ohgaki H (1997) Tumors associated with p53 germline mutations: a synopsis of 91 families. Am J Pathol 150:1-13

Kuperwasser C, Hurlbut GD, Kittrell FS, Dickinson ES, Laucirica R, et al. (2000) Development of spontaneous mammary tumors in $\mathrm{BALB} / \mathrm{c}$ p53 heterozygous mice. A model for Li-Fraumeni syndrome Am J Pathol 157:2151-2159

Lander E, Kruglyak L (1995) Genetic dissection of complex traits: guidelines for interpreting and reporting linkage results. Nat Genet 11:241-247

Malkin D, Li FP, Strong LC, Fraumeni JF Jr, Nelson CE, et al. (1990) Germ line p53 mutations in a familial syndrome of breast cancer, sarcomas, and other neoplasms. Science 250:1233-1238

Mira YLR, Zheng WL, Kuppumbatti YS, Rexer B, Jing Y, et al. (2000) Retinol conversion to retinoic acid is impaired in breast cancer cell lines relative to normal cells. J Cell Physiol 185:302-309

Murphy SM, Preble AM, Patel UK, O'Connell KL, Dias DP, et al. (2001) GCP5 and GCP6: two new members of the human gamma-tubulin complex. Mol Biol Cell 12:3340-3352

Rebbeck TR, Kantoff PW, Krithivas K, Neuhausen S, Blackwood MA, et al. (1999) Modification of BRCA1-associated breast cancer risk by the polymorphic androgen-receptor CAG repeat. Am J Hum Genet 64:1371-1377

Rexer BN, Zheng WL, Ong DE (2001) Retinoic acid biosynthesis by normal human breast epithelium is via aldehyde dehydrogenase 6, absent in MCF-7 cells. Cancer Res 61:7065-7070

Shepel LA, Lan H, Haag JD, Brasic GM, Gheen ME, et al. (1998) Genetic identification of multiple loci that control breast cancer susceptibility in the rat. Genetics 149:289-299

Srivastava S, Zou ZQ, Pirollo K, Blattner W, Chang EH (1990) Germline transmission of a mutated p53 gene in a cancer-prone family with Li-Fraumeni syndrome. Nature 348:747-749

Trkova M, Hladikova M, Kasal P, Goetz P, Sedlacek Z (2002) Is there anticipation in the age at onset of cancer in families with $\mathrm{Li}$ Fraumeni syndrome? J Hum Genet 47:381-386

Vitezica ZG, Elsen JM, Rupp R, Diaz C (2005) Using genotype probabilities in survival analysis: a scrapie case. Genet Sel Evol 37:403-415

Wingo PA, Ries LA, Rosenberg HM, Miller DS, Edwards BK (1998) Cancer incidence and mortality, 1973-1995: a report card for the U.S. Cancer 82:1197-1207

Woodage T, Basrai MA, Baxevanis AD, Hieter P, Collins FS (1997) Characterization of the CHD family of proteins. Proc Natl Acad Sci U S A 94:11472-11477 